\title{
SMOOTH EXTENDABILITY OF PROPER HOLOMORPHIC MAPPINGS
}

\author{
BY KLAS DIEDERICH AND JOHN ERIK FORNAESS
}

In [9] Ch. Fefferman proved that any biholomorphic mapping $f: \Omega_{1} \rightarrow \Omega_{2}$ between strictly pseudoconvex $C^{\infty}$-smooth domains in $\mathbf{C}^{n}$ extends smoothly to the boundary. Subsequently, the proof of this result has been simplified considerably by $S$. Webster $[14,15]$, E. Ligocka [12], St. Bell [1, 2]. And it was St. Bell who realized the importance of the following regularity condition of the Bergman projection for the proof of such extendability results:

DEFINITION. A domain $\Omega \subset \subset \mathbf{C}^{n}$ is said to satisfy condition $R$ for its Bergman projection operator $P$ if for any positive integer $s$ there is an integer $N$ such that $P$ is a bounded linear operator from $W_{0}^{s+N}(\Omega)$ to $H^{s}(\Omega)$.

(Here $W_{0}^{s}(\Omega)$ denotes as usual the closure of $C_{0}^{\infty}(\Omega)$ in the Sobolev s-Norm $\|\cdot\|_{s}$ with respect to the volume Lebesgue-measure on $\Omega$ and $H^{s}(\Omega)$ is the space of holomorphic functions on $\Omega$ with finite $\|\cdot\|_{s}$-norm.)

Since condition $R$ is a consequence of subelliptic estimates for the $\bar{\partial}$ Neumann problem, it is known to be satisfied for instance in the following cases:

(1) $\Omega$ strictly pseudoconvex, $C^{\infty}$-smooth (J. J. Kohn [10]);

(2) $\Omega$ pseudoconvex, $C^{\omega}$-smooth (J. J. Kohn [11], K. Diederich, J. E. Fornaess [6]).

The new methods allowed to generalize Fefferman's result. It is now known that a biholomorphic mapping $f: \Omega_{1} \rightarrow \Omega_{2}$ extends smoothly up to the boundary if $\Omega_{1}$ and $\Omega_{2}$ are $C^{\infty}$-smooth and, in addition, both satisfy condition $R$ [1] or both are pseudoconvex and at least one satisfies condition $R$ [2] .

The result which we wish to announce deals with the case of proper holomorphic mappings and is contained in the

Theorem. Let $\Omega_{1}, \Omega_{2} \subset \subset \mathrm{C}^{n}$ be $C^{\infty}$-smooth pseudoconvex domains and suppose that $\Omega_{1}$ satisfies condition $R$. Then any proper holomorphic mapping $f: \Omega_{1} \rightarrow \Omega_{2}$ extends smoothly up to the boundary.

For unbranched mappings $f$ this result is contained in K. Diederich and J. E. Fornaess [7]. This also includes the case of $\Omega_{1}, \Omega_{2}$ being strictly pseudoconvex and $f$ proper holomorphic since any such $f$ is necessarily unbranched, S. Pincuk [13]. Under different, more restrictive assumptions on $\Omega_{1}$ and $\Omega_{2}$ the result

Received by the editors January 6, 1982.

1980 Mathematics Subject Classification. Primary 32H99, 32F15.

(c) 1982 American Mathematical Society 0273-0979/82/0000-0296/\$02.25 
was obtained by S. Bell in [4 and 5]. More detailed information about the history will be given in the paper containing also all the proofs.

Our proof is based on the following transformation formula for the Bergman projection proved by S. Bell [3]:

Proposition 1. Let $f: \Omega_{1} \rightarrow \Omega_{2}$ be a proper holomorphic mapping between bounded domains in $\mathbf{C}^{n}$ and let $P_{i}$ denote the Bergman projection on $\Omega_{i}$. Then one has

$$
P_{1}\left(u \cdot((h \circ f))=u \cdot\left(P_{2}(h) \circ f\right)\right.
$$

for all $h \in L^{2}\left(\Omega_{2}\right)$. Here $u=\operatorname{det} f^{\prime}$.

As a consequence of this, S. Bell [3] obtains

Proposition 2. Under the assumptions of the theorem one has $u \cdot h \circ f \in$ $A^{\infty}\left(\Omega_{1}\right)$ for all $h \in A^{\infty}\left(\Omega_{2}\right)$, in particular $u \in A^{\infty}\left(\Omega_{1}\right)$.

The difficulty in deriving the theorem from this statement is to show that the functions $u \cdot h \circ f \in A^{\infty}\left(\Omega_{1}\right)$ can be divided by $u$ in $A^{\infty}\left(\Omega_{1}\right)$ even close to the cluster points of the branching locus $X$ of $f$ at $b \Omega_{1}$. This, certainly, would be hopeless if the Jacobian determinant $u$ could vanish to infinite order at such points. But we can show

LEMмA 1. In the situation of the theorem the Jacobian determinant $u$ of $f$ does not vanish to infinite order at any point of $\bar{\Omega}_{1}$.

For the proof we assume that $u$ does vanish to infinite order at $q \in b \Omega_{1}$ and take an arbitrary nontangential cone $K$ in $\Omega_{1}$ with vertex at $q$. The function

$$
u_{1}(z):=\prod_{\zeta \in f^{-1_{\circ}(z)}} u(\zeta) \text { for } \zeta \in \Omega_{1} \backslash X
$$

extends to a bounded holomorphic function on $\Omega_{1}$ which vanishes in $\Omega_{1}$ exactly on $X_{1}:=f^{-1} f(X)$ and at $q$ goes to zero with infinite order. There is a bounded holomorphic function $u_{2}$ on $\Omega_{2}$ with $u_{1}=u_{2} \circ f$ and $\left\{u_{2}=0\right\}=f\left(X_{1}\right)=X_{2}$. Notice, that on $\Omega_{2} \backslash X_{2}$ there are locally defined inverse mappings $F_{1}, \ldots, F_{m}$ of $f$, the Jacobian determinants of which are denoted by $U_{k}$. One has

$$
u_{2} \cdot \prod_{k=1}^{m} U_{k} \equiv 1
$$

Furthermore, it is well known that there are an $\eta>0$ and positive constants $c$, $C$ such that

$$
c \operatorname{dist}^{1 / \eta}\left(z ; b \Omega_{1}\right) \leqslant \operatorname{dist}\left(f(z), b \Omega_{2}\right) \leqslant C \operatorname{dist}^{\eta}\left(z, b \Omega_{1}\right)
$$

on $\Omega_{1}$. By considering for each fixed $k=1, \ldots, n$, the elementary symmetric functions of the $k$ th coordinate functions of $F_{j}, j=1, \ldots, m$, which are globally 
defined and bounded on $\Omega_{2}$, applying the Schwarz lemma to them and studying the zeros of the polynomial in one variable with these coefficients we can show using (2) that for large positive integers $N$ the preimage of a polynomial neighborhood of $X_{2}$ of the form

$$
V_{N}^{2}:=\left\{w \in \Omega_{2}: \operatorname{dist}\left(w, X_{2}\right)<\operatorname{dist}^{N}\left(w, b \Omega_{2}\right)\right\}
$$

is contained in a polynomial neighborhood $V_{M}^{1}$ of $X_{1}$ in $\Omega_{1}$ of the same form. Here $M<N$, but $M$ goes to infinity if $N$ does. Next by using the Blaschke condition for the zero set $X_{1}$ of $u_{1}$ one proves that for large $M$

$$
\lim _{r \rightarrow 0} \frac{A\left(V_{M}^{1} \cap K \cap B_{r}\right)}{A\left(K \cap B_{r}\right)}=0
$$

where $A()$ means euclidean volume and $B_{r}$ is the ball of radius $r$ around $q$. Therefore, there is a sequence $\left(z^{k}\right) \subset K, z^{k} \rightarrow q$, such that $\Omega_{2} \backslash X_{2}$ contains for each $k$, a relatively "large" ball around $f\left(z^{k}\right)$. This allows us to show that all $U_{j}\left(f\left(z^{k}\right)\right)$ have to be small compared to a fixed negative power of

$$
\operatorname{dist}\left(f\left(z^{k}\right), b \Omega_{2}\right) \text {. }
$$

Because of (1) and (2) we obtain that $u_{1}\left(z^{k}\right)$ stays above a certain fixed power of $\operatorname{dist}\left(z^{k}, b \Omega_{1}\right)$. This contradicts our assumption.

The next step is the division by $u$. We show

LEMMA 2. Let $h$ be a bounded holomorphic function on $\Omega_{1}$ such that $u \cdot h^{N} \in A^{\infty}\left(\Omega_{1}\right)$ for all positive integers $N$, then $h \in A^{\infty}\left(\Omega_{1}\right)$.

This is proved by a rather technical inductive procedure. We will, therefore indicate here only how the continuity of $h$ follows. We fix a point $q \in b \Omega_{1}$ and a generic transverse complex line $H$ through $q$ such that $u \mid H \cap \Omega_{1}$ vanishes at $q$ only to finite order $k$. We can assume that $q=0$ and

$$
H=\left\{z_{2}=\cdots=z_{n}=0\right\} .
$$

For $p \in b \Omega_{1}$ near 0 we denote by $H(p)$ the complex line through $p$ parallel to $H$. It is easy to see that $h \mid \Omega_{1} \cap H_{p}$ is $C^{\infty}$ up to $b \Omega_{1} \cap H_{p}$ near 0 . This gives us an extension of $h$ to $b \Omega_{1}$ near 0 . We may assume that $h(0)=0$. We denote

$$
g^{(s)}:=\partial^{s} g / \partial z_{1}^{s}
$$

and use the following observation.

LEMmA 3. For each $k$ there exists a sequence of polynomials $P_{j}, j=1,2$, $3, \ldots$, in $u$ and $h$ and their derivatives, such that for all $N>k$

$$
\left(u h^{N}\right)^{(k)}=u^{(k)} h^{N}+\left(\sum_{j=1}^{k} N^{j} P_{j}\right) h^{N-k} .
$$


The proof goes by induction over $k$ and is straightforward. Let us now assume that there is a sequence $S=\left(p_{s}\right) \subset \Omega_{1}, p_{s} \rightarrow 0$, such that $h\left(p_{s}\right) \rightarrow$ $\hat{\gamma} \neq 0$. For $N>k$ we have

$$
\left(u h^{N}\right)^{(k)} \rightarrow 0 \text { on } S,
$$

since this function is in $A^{\infty}$ and its restriction to $H(0) \cap \Omega_{1}$ goes to zero at 0 . Therefore, we get from (4)

$$
u^{(k)} h^{k}+\left(\sum_{j=1}^{k} N^{j} P_{j}\right) \rightarrow 0 \text { on } S
$$

Next we prove inductively for $0 \leqslant k \leqslant k$ :

$\left(\mathrm{A}_{\kappa}\right)$ There are coefficients $a_{j, s}^{(\kappa)}, j=1, \ldots, \kappa, s=1,2,3, \ldots$, such that for all $N>k$

$$
u^{(k)} h^{k}\left(p_{s}\right)+\sum_{j=1}^{\kappa} N^{j} a_{j, s}^{(\kappa)} \rightarrow 0 \quad \text { as } s \rightarrow \infty .
$$

For $\kappa=k$ the choice $a_{j, s}^{(k)}:=P_{j}\left(p_{s}\right)$ satisfies $\left(\mathrm{A}_{k}\right)$ because of (6). If $\left(\mathrm{A}_{\kappa}\right)$ for some $1 \leqslant k \leqslant k$ has been reached, one may put

$$
a_{j, s}^{(\kappa-1)}:=a_{j, s}^{(\kappa)}\left(1-2^{j} 2^{-\kappa}\right)\left(1-2^{-\kappa}\right)^{-1} .
$$

That this satisfies $\left(A_{\kappa-1}\right)$ follows from the fact that

$\left(1-2^{-\kappa}\right)^{-1}\left[u^{(k)} h^{k}\left(p_{s}\right)+\sum_{j=1}^{\kappa} N^{j} a_{j, s}^{(\kappa)}-2^{-\kappa}\left(u^{(k)} h^{k}\left(p_{s}\right)+\sum_{j=1}^{\kappa}(2 N)^{j} a_{j, s}^{(\kappa)}\right)\right] \rightarrow 0$ as $s \rightarrow \infty$. The statement $\left(\mathrm{A}_{0}\right)$ now gives $u^{(k)} h^{k} \rightarrow 0$ on $S$, a contradiction to $h \rightarrow \hat{\gamma} \neq 0$ on $S$.

An easy consequence of our theorem is the following statement. An independent and simpler proof of it has been given in [8].

Corollary. Let $f: \Omega_{1} \rightarrow \Omega_{2}$ be proper holomorphic, $\Omega_{1}, \Omega_{2} C^{\infty}$. smooth and pseudoconvex. Suppose that $\Omega_{1}$ satisfies condition $R\left(e . g . \Omega_{1}\right.$ strictly pseudoconvex). Then the branching locus of $f$ does not cluster at any strictly pseudoconvex boundary point. In particular, $f$ is unbranched if the $(2 n-3)$-dimensional Hausdorff measure of the set of weakly pseudoconvex boundary points of $\Omega_{1}$ is zero.

\section{REFERENCES}

1. S. Bell and E. Ligocka, $A$ simplification and extension of Fefferman's theorem on biholomorphic mappings, Invent. Math. 57 (1980), 283-289. 103-113.

2. S. Bell. Biholomorphic mappings and the J-problem, Ann. of Math. (2) 114 (1981),

3. Proper holomorphic mappings and the Bergman projection, Duke Math. J. 48 (1981), 167-175. print (1981). 
5. S. Bell, Analytic hypoellipticity of the $\bar{\partial}$-Neumann problem and extendability of holomorphic mappings, Preprint (1981).

6. K. Diederich and J. E. Fornaess, Pseudoconvex domains with real-analytic boundary, Ann. of Math. (2) 107 (1978), 371-384.

7. - A remark on a paper of S. Bell, Manuscripta Math. 34 (1981), 31-44. (1981).

8. - Proper holomorphic images of strictly pseudoconvex domains, Preprint

9. Ch. Fefferman, The Bergman kernel and biholomorphic mappings of pseudoconvex domains, Invent. Math. 26 (1974), 1-65.

10. J. J. Kohn, Harmonic integrals on strongly pseudoconvex manifolds. I and II, Ann. of Math. (2) 78 (1963), $112-148 ; 79$ (1964), 450-472.

11. Subellipticity of the $\bar{\partial}$-Neumann problem on pseudoconvex domains: sufficient conditions, Acta Math. 142 (1979), 79-122.

12. E. Ligocka, How to prove Fefferman's theorem without use of differential geometry, Ann. Polon. Math.

13. S. Pincuk, Proper holomorphic mappings between strongly pseudoconvex domains,

Dokl. Akad. Nauk SSSR 241 (1978), 30-33.

14. S. Webster, On the proof of boundary smoothness of biholomorphic mappings, Preprint (1978).

15. Biholomorphic mappings and the Bergman kernel off the diagonal, Invent. Math. 51 (1979), 155-169.

UNIVERSITÄT WUPPERTAL, MATHEMATIK, GAUßSTR. 20, D-5600 WUPPERTAL 1, WEST GERMANY NORWAY

MATEMATISK INSTITUTT, UNIVERSITETET I OSLO, BLINDERN, OSLO 3, 